\title{
Recent Progress in Mathematical Diffraction
}

\author{
U. GRIMM ${ }^{a}$ AND M. BAAKE ${ }^{b}$ \\ ${ }^{a}$ Department of Mathematics and Statistics, The Open University, Walton Hall, Milton Keynes MK7 6AA, UK \\ ${ }^{b}$ Fakultät für Mathematik, Universität Bielefeld, Postfach 100131, 33501 Bielefeld, Germany
}

\begin{abstract}
A brief summary of recent developments in mathematical diffraction theory is given. Particular emphasis is placed on systems with aperiodic order and continuous spectral components. We restrict ourselves to some key results and refer to the literature for further details.
\end{abstract}

DOI: $10.12693 /$ APhysPolA.126.474

PACS: 61.05.cc, 61.43.-j, 61.44.Br

\section{Introduction}

Diffraction methods [1] continue to provide the main tool for the structure analysis of solids. The corresponding inverse problem of determining a structure from its diffraction is difficult and, in general, does not define a structure uniquely. Kinematic diffraction, an approximation that is reasonable for X-ray diffraction where multiple scattering effects can be neglected, is well suited for a mathematical approach via measures. Measures are generalisations of the classic concept of the Lebesgue measure used in volume integration and provide a natural mathematical concept to quantify the distribution of matter in space as well as the distribution of scattering intensity. This mathematical approach to diffraction was pioneered by Hof [2] and has substantially been developed since the discovery of quasicrystals required an extension of the methods used to compute the diffraction of perfectly periodic crystals.

The need for further insight emerged from the question of which distributions of matter, beyond perfectly periodic crystals, lead to pure point diffraction patterns, hence to diffraction patterns comprising sharp Bragg peaks only. More recently, it has become apparent that one also has to study continuous diffraction in more detail, with a careful analysis of the different types (singular and absolutely continuous) of diffuse scattering involved. This is both of interest from a mathematical point of view, since the diffraction spectrum is closely related to the dynamical spectrum of the associated dynamical system [3], and from an experimental point of view, where diffuse scattering including candidates for singular continuous scattering are observed [4].

In this brief account of a tutorial review, we summarise key results, putting particular emphasis on the analysis of non-periodic structures. Following the presentation in our recent review articles $[5,6]$, general results are introduced and discussed on the basis of various characteristic examples, with minimal use of formal arguments or proofs. For details and more background material, we refer to the comprehensive treatment in [7].

\section{Diffraction measure}

We consider the diffraction measure mainly for Delone sets $\Lambda \subset \mathbb{R}^{d}$, which are point sets where the points neither get arbitrarily close nor so sparse that they accommodate arbitrarily large empty balls. Define the corresponding weighted Dirac comb

$$
\omega=w \delta_{\Lambda}=\sum_{x \in \Lambda} w(x) \delta_{x},
$$

where $\delta_{\Lambda}=\sum_{x \in \Lambda} \delta_{x}$ denotes the uniform comb of point scatterers $\delta_{x}$ at all positions $x \in \Lambda$, and where $w(x) \in \mathbb{C}$ represents the scattering weight at position $x$. In most cases we are interested in (and all that appear below), the function $w$ is such that $\omega$ is a translation bounded measure on $\mathbb{R}^{d}$. For instance, if $\Lambda$ is Delone, it suffices if $w$ is a bounded function.

The natural autocorrelation measure of $\omega$ is defined as the limit (provided it exists)

$$
\gamma=\gamma_{\omega}=\omega \circledast \widetilde{\omega}:=\lim _{R \rightarrow \infty} \frac{\left.\omega\right|_{R} * \widetilde{\left.\omega\right|_{R}}}{\operatorname{vol}\left(B_{R}\right)},
$$

where $\left.\omega\right|_{R}$ denotes the restriction of $\omega$ to the open ball $B_{R}$ of radius $R$ around $0 \in \mathbb{R}^{d}$, and $\widetilde{\mu}$ is the 'flippedover' version of a measure $\mu$ defined by $\widetilde{\mu}(g)=\overline{\mu(\widetilde{g})}$ for any measurable function $g$, where $\widetilde{g}(x)=\overline{g(-x)}$. The operation $\circledast$ (also known as the Eberlein convolution) is a volume-averaged analogue of the ordinary convolution $*$ of measures.

Assuming that the autocorrelation measure $\gamma$ of $\omega$ exists, its Fourier transform $\widehat{\gamma}$ is a well-defined translation bounded, positive measure, called the diffraction measure of $\omega$. It describes the kinematic scattering intensity observed in an experiment. The measure $\widehat{\gamma}$ has a unique decomposition

$$
\widehat{\gamma}=\widehat{\gamma}_{\mathrm{pp}}+\widehat{\gamma}_{\mathrm{sc}}+\widehat{\gamma}_{\mathrm{ac}}
$$

into a pure point part (comprising the Bragg peaks, of which there are at most countably many), an (with respect to the Lebesgue measure) absolutely continuous part (the diffuse background scattering, which has a locally integrable density) and a singular continuous part (which comprises anything that remains).

\section{Perfect crystals}

A perfect (infinite) crystal in $\mathbb{R}^{d}$ corresponds to a lattice-periodic discrete structure. Denoting its lattice of periods by $\Gamma \subset \mathbb{R}^{d}$ and specifying the decoration of a fundamental domain of $\Gamma$ with scatterers by a finite 
measure $\mu$, one obtains the crystallographic measure

$$
\omega=\mu * \delta_{\Gamma},
$$

with autocorrelation $\gamma=\operatorname{dens}(\Gamma)(\mu * \widetilde{\mu}) * \delta_{\Gamma}$. The Fourier transform of lattice-periodic measures can be calculated using Poisson's summation formula

$$
\widehat{\delta_{\Gamma}}=\operatorname{dens}(\Gamma) \delta_{\Gamma^{*}},
$$

where $\Gamma^{*}$ denotes the dual or reciprocal lattice of $\Gamma$, defined by

$$
\Gamma^{*}=\left\{x \in \mathbb{R}^{d} \mid\langle x \mid y\rangle \in \mathbb{Z} \text { for all } y \in \Gamma\right\} .
$$

Note that our convention for the Fourier transform is $\widehat{\phi}(k):=\int_{\mathbb{R}^{d}} e^{-2 \pi i\langle k \mid x\rangle} \phi(x) \mathrm{d} x$, where $k, x \in \mathbb{R}^{d}$ with scalar product $\langle k \mid x\rangle$. The diffraction measure of the crystallographic measure of Eq. (4) is then obtained as

$$
\widehat{\gamma}=(\operatorname{dens}(\Gamma))^{2}|\widehat{\mu}|^{2} \delta_{\Gamma^{*}},
$$

which is a pure point measure supported on the reciprocal lattice $\Gamma^{*}$, with scattering intensities that can be calculated from the (continuous) Fourier transform of the finite measure $\mu$.

As a simple example, consider a $\mathbb{Z}^{2}$-periodic system with two scatterers in a unit cell, one of unit scattering strength at position $(0,0)$ and one of scattering strength $\alpha \in \mathbb{C}$ at position $(a, b)$. The corresponding weighted Dirac comb is $\omega=\mu * \delta_{\mathbb{Z}^{2}}$ with $\mu=\delta_{(0,0)}+\alpha \delta_{(a, b)}$. The diffraction measure is $\widehat{\gamma_{\omega}}=|\widehat{\varrho}|^{2} \delta_{\mathbb{Z}^{2}}$ (note that the lattice $\mathbb{Z}^{2}$ is self-dual) with diffraction intensities

$$
I\left(k_{1}, k_{2}\right)=|\widehat{\varrho}|^{2}\left(k_{1}, k_{2}\right)=\left|1+\alpha \mathrm{e}^{-2 \pi \mathrm{i}\left(k_{1} a+k_{2} b\right)}\right|^{2},
$$

which are evaluated at the points $\left(k_{1}, k_{2}\right) \in \mathbb{Z}^{2}$ of the reciprocal lattice. Note that, in general, the intensity distribution is not periodic, while the location set of the Bragg peaks is (provided there are no extinctions).

\section{Euclidean model sets}

Model sets (or cut and project sets) arise from projections from a lattice $\mathcal{L}$ in a higher-dimensional space. The general setting for a Euclidean model set is encoded in the cut and project scheme (CPS)

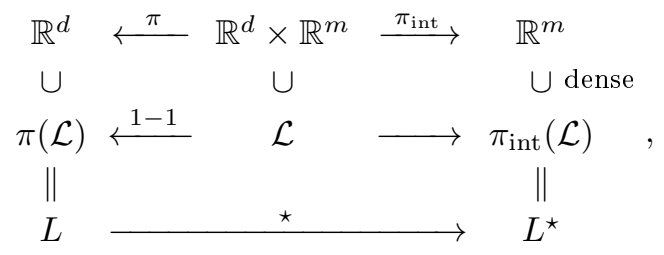

where $\mathbb{R}^{d}$ is the physical and $\mathbb{R}^{m}$ the internal space, and $\mathcal{L} \subset \mathbb{R}^{d+m}$. The associated natural projections are denoted by $\pi$ and $\pi_{\text {int }}$. The bijectivity of the projection on $L=\pi(\mathcal{L}) \subset \mathbb{R}^{d}$ and the denseness of $L^{\star}=\pi_{\text {int }}(\mathcal{L}) \subset \mathbb{R}^{m}$ ensure that the $\star$-map $x \mapsto x^{\star}$ is well-defined on $L$ and the model is free of redundance.

For a fixed CPS and a window $W \subset \mathbb{R}^{m}$, the set

$$
\Lambda=\left\{x \in L \mid x^{\star} \in W\right\}
$$

is called a model set (or cut and project set). As long as the window is sufficiently well behaved, see [7], Sec. 9.4 for the details, the corresponding Dirac comb $\delta_{\Lambda}$ has the pure point diffraction measure

$$
\widehat{\gamma}=\sum_{k \in L^{\circledast}}|A(k)|^{2} \delta_{k},
$$

which is supported on the Fourier module $L^{\circledast}=\pi\left(\mathcal{L}^{*}\right)$, the projection of the higher-dimensional dual lattice. The diffraction amplitudes $A(k)$ are explicitly given by

$$
A(k)=\frac{\operatorname{dens}(\Lambda)}{\operatorname{vol}(W)} \widehat{1_{W}}\left(-k^{\star}\right),
$$

where $1_{W}$ denotes the characteristic function of the window $W$, and $\star$ is the star-map of the CPS. So, the calculation of diffraction intensities essentially requires the Fourier transform of the characteristic function of the window $W$, which can be done explicitly for many examples with polygonal or spherical windows. Clearly, the symmetry of the window $W$ manifests itself in the symmetry of the diffraction intensities; see [7] for examples.

Note that, while $\widehat{\gamma}$ is a pure point measure, it is supported on the projection of the entire reciprocal lattice, which (in general) results in a dense point set in $\mathbb{R}^{d}$. Nevertheless, the total intensity scattered into any region of space, which corresponds to summing up infinitely many intensities of peaks in that region, always remains finite. Restricting to peaks with intensities above any given threshold thus produces a discrete pattern of peaks.



Fig. 1. Sketch of the diffraction of the Fibonacci point set for $0 \leq k \leq 20$. A peak of this pure point measure is represented as a line with a height that equals its intensity. All peaks with at least $1 / 1000$ of the central intensity $I(0)=(\tau+1) / 5 \approx 0.5236$ are included.

As a one-dimensional example, consider the Fibonacci point set which is obtained from a CPS with planar lattice $\mathcal{L}=\left\{\left(x, x^{\star}\right) \mid x \in \mathbb{Z}[\tau]\right\} \subset \mathbb{R}^{2}$. Here, $\tau=(1+\sqrt{5}) / 2$ is the golden number, $L=\mathbb{Z}[\tau]=\{a+b \tau \mid a, b \in \mathbb{Z}\}$ and the star-map acts as algebraic conjugation $(a+b \tau)^{\star}=$ $a+b(1-\tau)$. The window is $W=(-1, \tau-1]$ (chosen as half-open to avoid singular cases), which produces a regular model set of density $\tau / \sqrt{5}=(\tau+2) / 5$. The Fourier module is $L^{\circledast}=L / \sqrt{5}$, and the diffraction intensity for $k \in L / \sqrt{5}$ is obtained as 


$$
I(k)=\left(\frac{\tau}{\sqrt{5}} \operatorname{sinc}\left(\pi \tau k^{\star}\right)\right)^{2},
$$

where $\operatorname{sinc}(x)=\sin (x) / x$; a sketch of the diffraction patterns in shown in Fig. 1. The intensity function $I(k)$ vanishes on $L^{\circledast}$ if and only if $\tau k^{\star} \in \mathbb{Z} \backslash\{0\}$. This corresponds to $k=\ell \tau$ with $\ell \in \mathbb{Z} \backslash\{0\}$, and the Bragg peaks at these positions are extinct. Another apparent feature of Fig. 1 is the presence of series of peaks with increasing intensity. These series appear at $\tau$-scaled positions, and are a consequence of the fact that $\left|\left(\tau^{m}\right)^{\star}\right|=|1-\tau|^{m}<1$, which by Eq. (11) implies that, for any $k$ with $I(k)>0$, one has $I\left(\tau^{m} k\right) \rightarrow I(0)$ for $m \rightarrow \infty$. In particular, one can clearly see this phenomenon for the peaks at $\tau^{i} / \sqrt{5}$ with $0 \leq i \leq 7$, where $\tau^{5} / \sqrt{5} \approx 4.96$.

\section{Singular continuous diffraction}

Singular continuous measures are rather strange, as they give no weight to any single point, but are still concentrated to an uncountable set of zero Lebesgue measure. A well-known example is the 'Devil's staircase', which is the distribution function of the probability measure for the classic middle-thirds Cantor set, which is constant almost everywhere. Singular continuous diffraction can occur in experiment [4] and in realistic models, and thus should not be disregarded.

The paradigm for singular continuous diffraction is the Thue-Morse (TM) system [8-10], based on the binary substitution rule $1 \mapsto 1 \overline{1}, \overline{1} \mapsto \overline{1} 1$. The recursion $v^{(n+1)}=v^{(n)} \overline{v^{(n)}}$ with initial condition $v^{(0)}=1$ clearly converges to the one-sided fixed point $v=v_{0} v_{1} v_{2} \ldots$ of the TM substitution. The exponential sum

$$
g_{n}(k)=\sum_{\ell=0}^{2^{n}-1} v_{\ell} \mathrm{e}^{-2 \pi \mathrm{i} k \ell}
$$

is the Fourier transform of the weighted Dirac comb $\omega_{n}=\sum_{\ell=0}^{2^{n}-1} v_{\ell} \delta_{\ell}$ for the (finite) word $v^{(n)}$, where we identify $1=-1$. The exponential sum of Eq. (12) satisfies the recursion

$$
g_{n+1}(k)=\left(1-\mathrm{e}^{-2 \pi \mathrm{i} k 2^{n}}\right) g_{n}(k)
$$

for $n \geq 0$, with $g_{0}(k)=1$. With $\widehat{\gamma}=\lim _{n \rightarrow \infty}\left|g_{n}\right|^{2} / 2^{n}$, which converges (as a measure) in the vague topology, the diffraction measure of the TM system can now be represented as a Riesz product [9]

$$
\widehat{\gamma}=\prod_{n \geq 0}\left(1-\cos \left(2^{n+1} \pi k\right)\right) .
$$

The corresponding distribution function $F(k):=\widehat{\gamma}([0, k])$ is continuous. Moreover, it possesses a uniformly converging Fourier series $[11,12]$; it is shown in Fig. 2. For scaling properties of the Thue-Morse spectrum, we refer to [13] and references therein.

While singular continuous spectra may appear rather special, they are in fact quite common; compare [14] for a corresponding concrete result from the spectral theory of the Schrödinger operators. The TM approach can be generalised to show that large classes of bijective bi-

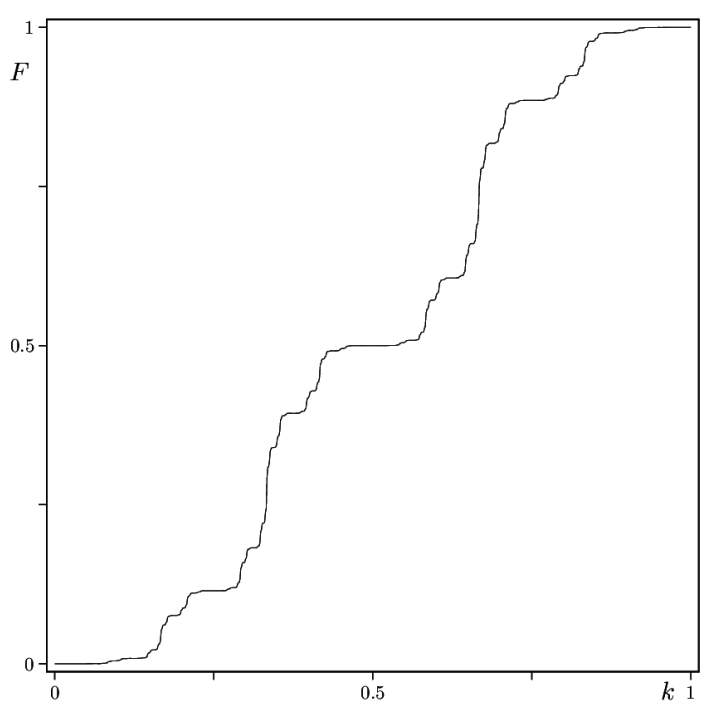

Fig. 2. The (strictly increasing) distribution function $F(k)$ of the TM diffraction measure of Eq. (13).

nary substitution systems have purely singular continuous diffraction [15], which includes higher-dimensional systems such as the squiral tiling [16].

\section{Absolutely continuous diffraction}

Usually, absolutely continuous diffraction is connected with disorder [17]. Indeed, randomness often gives rise to continuous components in the diffraction, though it is important to remember that there are also 'ordered' structures that may lead to absolutely continuous spectra. The probably best known example is the Rudin-Shapiro (RS) system, which can be defined by the sequence of weights $w_{n} \in\{ \pm 1\}$ with initial conditions $w_{-1}=-1$, $w_{0}=1$, and the recursion

$$
w_{4 n+\ell}= \begin{cases}w_{n}, & \text { for } \ell \in\{0,1\}, \\ (-1)^{n+\ell} w_{n}, & \text { for } \ell \in\{2,3\} .\end{cases}
$$

The corresponding Dirac comb $\omega=\sum_{n \in \mathbb{Z}} w_{n} \delta_{n}$ has the autocorrelation measure $\gamma=\delta_{0}$, and hence the Lebesgue measure as diffraction measure. In other words, the diffraction is completely 'featureless', with constant intensity for all $k \in \mathbb{R}$.

A simple random system that shares this property is the binary Bernoulli chain, where the weight at each position $n \in \mathbb{Z}$ is chosen independently to be either 1 or -1 , with equal probability $p=1 / 2$. These two systems are thus homometric. More surprisingly, as shown in [18], this example can be generalised to an entire family of homometric systems of the form

$$
\omega_{p}=\sum_{n \in \mathbb{Z}} w_{n} X_{n} \delta_{n}
$$

where $\left(w_{n}\right)_{n \in \mathbb{Z}}$ is the binary RS system defined in Eq. (14), and where $\left(X_{n}\right)_{n \in \mathbb{Z}}$ is an i.i.d. family of random numbers taking values 1 and -1 with probabilities 
$p$ and $1-p$. This example should serve as a warning concerning the inverse problem of diffraction - in general, this is not unique, and may not even be sensitive to 'order' or 'disorder', for instance in the sense of entropy (as in this case).

While some progress has been made to understand the diffraction of systems with stochastic disorder (see [5-7] and references therein), systems with correlated disorder as well as random tilings, which are of particular interest in the context of quasicrystals, are generally difficult to treat. As a simple example, consider the one-dimensional Fibonacci random tiling, which is the ensemble of all tilings of $\mathbb{R}$ with two prototiles (one interval of length $\tau$ and one of length 1 ), chosen with probabilities $p=\tau^{-1}$ and $1-p$, respectively. For this system, you find that, almost surely, the diffraction measure satisfies

$$
\widehat{\gamma}=\left(\frac{\tau+2}{5}\right)^{2} \delta_{0}+h(k) \lambda,
$$

where $\lambda$ denotes the Lebesgue measure and where the corresponding Radon-Nikodym density $h$ is given by

$$
h(k)=\frac{\tau+2}{5} \frac{\sin ^{2}(\pi k / \tau)}{\tau^{2} \sin ^{2}(\pi k \tau)+\tau \sin ^{2}(\pi k)-\sin ^{2}(\pi k / \tau)} .
$$

Beside the trivial Bragg peak at $k=0$, the diffraction is absolutely continuous. The function $h$ is shown in Fig. 3; while it is smooth, it still displays a spiky structure resembling the pure point diffraction of the perfect system shown in Fig. 1.

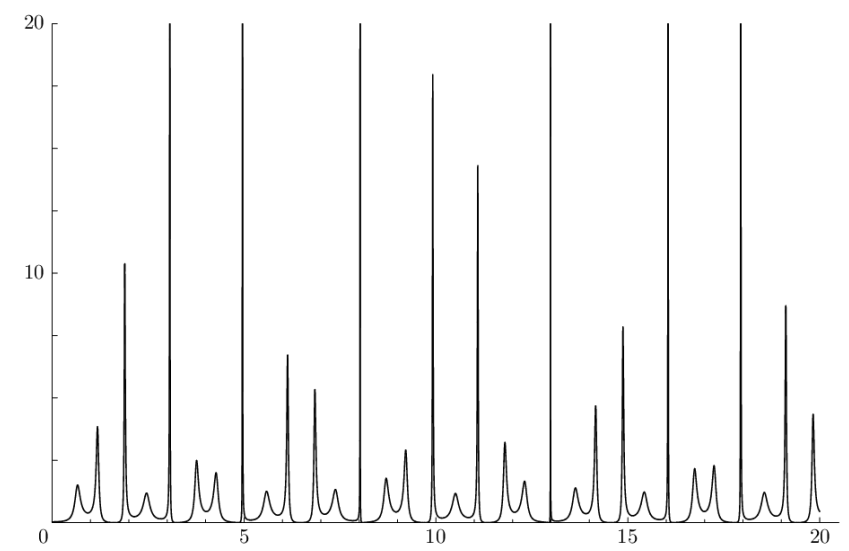

Fig. 3. The absolutely continuous part of the diffraction pattern of a Fibonacci random tiling, for the same range of the wave number $k$ as in Fig. 1.

An alternative way of introducing disorder into a Fibonacci system (or more generally, into a substitution system) is obtained by mixing different inflation rules $l o-$ cally $[19,20]$. This produces interesting tiling ensembles of positive entropy, where under certain conditions (as in the case of the noble means substitutions) all realisations are Meyer sets [20]. Consequently, they possess non-trivial Bragg diffraction (in line with general results by Strungaru [21]), but in addition show absolutely continuous diffraction; see [20, 22] for details.

\section{Outlook}

The discovery of quasicrystals in 1982 was based on its unusual diffraction pattern, displaying crystallographically 'forbidden' icosahedral symmetry. Such diffraction patterns are by now well understood, in the sense that it has been shown rigorously that, with some modest assumptions on the window, the diffraction measure of mathematical quasicrystals (model sets) are pure point measures, so comprise the Bragg peaks only. It remains to achieve a better understanding of the cases with continuous diffraction, which carries important information on the structure of a system as well, not just in the case where the Bragg peaks are absent. While progress has been made, and some explicit examples of singular and absolutely continuous spectra have become accessible, see [23] and references therein, there are still important systems that require further research. This includes proper random tilings in two and more dimensions, as well as substitution and inflation-based structures such as non-Pisot substitution systems or pinwheel-type tilings with continuous symmetries.

From a mathematical point of view, the relation between diffraction spectra and dynamical spectra of the associated dynamical system (under translation action) is now much better understood; see [3] and references therein for recent developments. Also, with methods from the theory of (stochastic) point processes, the inverse problem of structure determination from a pure point (or Bragg) diffraction spectrum has been understood in an abstract setting in rather large generality [24]; see also [25, 26] for additional examples in this context. The corresponding problem for general (mixed) spectrum is still open, and appears to be rather challenging.

\section{Acknowledgments}

This work was supported by the German Research Foundation (DFG), within the CRC 701.

\section{References}

[1] J.M. Cowley, Diffraction Physics, 3rd ed., NorthHolland, Amsterdam 1995.

[2] A. Hof, Commun. Math. Phys. 169, 25 (1995).

[3] M. Baake, D. Lenz, A.C.D. van Enter, arXiv:1307.7518, Erg. Th. \& Dyn. Syst., in press.

[4] R.L. Withers, Z. Kristallogr. 220, 1027 (2005).

[5] M. Baake, U. Grimm, Z. Kristallogr. 226, 711 (2011).

[6] M. Baake, U. Grimm, Chem. Soc. Rev. 41, 6821 (2012).

[7] M. Baake, U. Grimm, Aperiodic Order, Vol. 1, Cambridge University Press, Cambridge 2013.

[8] S. Kakutani, in: Proc. 6th Berkeley Symp. on Math. Statistics, and Probability, Eds.: L.M. LeCam, J. Neyman, E.L. Scott, Univ. of California Press, Berkeley 1972, p. 319. 
[9] M. Queffélec, Substitution Dynamical Systems Spectral Analysis, LNM 1294, 2nd ed., Springer, Berlin 2010.

[10] A.C.D. van Enter, J. Miȩkisz, J. Stat. Phys. 66, 1147 (1992).

[11] M. Baake, F. Gähler, U. Grimm, J. Math. Phys. 53, 032701 (2012).

[12] M. Baake, U. Grimm, J. Phys. A: Math. Theor. 41, 422001 (2008).

[13] M. Baake, U. Grimm, J. Nilsson, Acta Phys. Pol. A 126, 431 (2014).

[14] O. Knill, Discrete Contin. Dyn. Syst. 4, 33 (1998).

[15] N.P. Frank, Topol. Appl. 152, 44 (2005).

[16] M. Baake, U. Grimm, arXiv:1205.1384, Erg. Th. \& Dynam. Syst., in press.

[17] M. Baake, D. Lenz, C. Richard, Lett. Math. Phys. 82, 61 (2007)
[18] M. Baake, U. Grimm, Phys. Rev. B 79, 020203(R) (2009); erratum ibid. 80, 029903(E) (2009).

[19] C. Godrèche, J.M. Luck, J. Stat. Phys. 55, 1 (1989).

[20] M. Baake, M. Moll, in: Aperiodic Crystals, Eds. S. Schmidt, R.L. Withers, R. Lifshitz, Springer, Dordrecht 2013, p. 19, arXiv:1210.3462.

[21] N. Strungaru, Discr. Comput. Geom. 33, 483 (2005).

[22] M. Moll, Acta Phys. Pol. A 126, 535 (2014).

[23] M. Baake, M. Birkner, R.V. Moody, Commun. Math. Phys. 293, 611 (2010).

[24] D. Lenz, R.V. Moody, preprint arXiv:1111.3617, 2011.

[25] V. Terauds, J. Stat. Phys. 152, 954 (2013).

[26] V. Terauds, M. Baake, in: Aperiodic Crystals, Eds. S. Schmidt, R.L. Withers, R. Lifshitz, Springer, Dordrecht 2013, p. 35, arXiv:1210.3460. 